\title{
Hipertensión arterial: estrategias para derrotar al enemigo en Colombia
}

\author{
Arterial hypertension: strategies to defeat the enemy in Colombia \\ Javier Moreno-Cortés* \\ Servicio de Hipertensión Arterial, Sociedad Colombiana de Cardiología y Cirugía Cardiovascular, Bogotá, Colombia
}

La hipertensión arterial, ampliamente discutida en diferentes escenarios y textos académicos, es sin duda una situación de salud pública con impacto personal, social, laboral, económico y político de dimensiones importantes. Como bien hemos entendido a lo largo ya de varios años, la principal causa de muerte en el mundo es sin lugar a duda la enfermedad cardiovascular, siendo la hipertensión arterial el principal factor de riesgo para desarrollar estas situaciones clínicas, dada su carga atribuible en términos de morbilidad y mortalidad ${ }^{1}$, ubicándose además como la principal causa de muerte prematura en el mundo. Se estima que en el mundo actualmente hay 1130 millones de personas con hipertensión arterial, como reporta la Organización Mundial de la Salud (OMS), y la mayoría de ellos viven en países con ingresos medianos y bajos ${ }^{2}$.

El incremento de la presión arterial juega un rol protagónico en la génesis de estas enfermedades cardiovasculares y de la enfermedad renal crónica, con una evidente progresión de casos registrados desde hace ya varios años, y la estimación de un mayor aumento en sus cifras es una preocupación de ámbito mundial por todo el impacto en los diferentes escenarios de la dinámica de salud que se aproxima ${ }^{2}$, todo esto en un contexto alarmante, dado que las cifras estimadas de control o logro de metas de esta situación no superan el $30 \%$ en los diferentes registros mundiales ${ }^{3,4}$.

Son muchas las estrategias que se han planteado en todo el mundo, en cada una de sus naciones y en todos los escenarios académicos y políticos, para desarrollar planes que de manera pronta puedan mitigar los devastadores efectos de una situación clínica que a veces parece fuera de control y que puede poner en jaque a muchos de nuestros sistemas de salud, debido a la alta carga de enfermedades de alto costo que esta situación trae como consecuencia. El objetivo fundamental de ellas es desarrollar políticas conjuntas de todos los actores del sistema de salud involucrados en todo el proceso de crecimiento y desarrollo del individuo, desde su nacimiento hasta su edad anciana, desde un punto de vista individual y de población, encaminadas a intervenir y evitar la progresión de cada una de las etapas de la evolución natural de la hipertensión arterial, incluyendo umbrales de choque para modificación temprana, y asignación de roles personales, estatales y sociales para que en un trabajo conjunto se contenga y modifique el efecto no deseado ${ }^{5}$. A partir del año 2016, la OMS y la Organización Panamericana de la Salud implementan una estrategia global, denominada HEARTS, en la cual se incluyen la detección y el tratamiento de la hipertensión arterial dentro de un paquete de múltiples herramientas encaminadas a disminuir la mortalidad por enfermedad cardiovascular.

Actualmente, por la situación que atravesamos de manera global a causa del SARS-CoV-2, hemos visto cómo en la poblacional se ha generado una consciencia adherida con respecto a lo que implica la palabra 
«pandemia» y cómo una situación clínica puede afectarnos de manera conjunta; además, se ha logrado transmitir un mensaje categórico con respecto a la importancia de mantener bajo control aquellos factores de riesgo que tanto hemos explicado en los escenarios de atención a nuestros pacientes, pues en el contexto de otras enfermedades pueden cambiar el curso clínico favorable esperado. De igual manera, hemos aprendido la importancia en otros escenarios de perseverar en las metas de control y la relevancia que tiene mantener e iniciar los tratamientos adecuados en estos factores de enfermedad cardiovascular.

Un ejemplo de estas iniciativas globales se ha desarrollado en la Sociedad Colombiana de Cardiología y Cirugía Cardiovascular, y su capítulo de hipertensión arterial, en donde se han planteado puntos importantes en secuencia con el ánimo de incrementar el conocimiento del personal de salud y la unidad de criterios para llevar una línea uniforme de trabajo en la atención del paciente hipertenso, a través de la realización de consensos, cursos de expertos de hipertensión arterial, talleres académicos. Entre estas, quizá la mas importante es la desarrollada con el apoyo de la World Heart Federation (WHF) dentro de su proyecto de mapas de ruta (RoadMap), que busca incrementar la detección de la población hipertensa, que el paciente hipertenso sepa que lo es y lo que esto implica, y que esté en tratamiento temprano para así lograr un control adecuado y mantener la adherencia a las terapias.

Dentro de lo propuesto por la WHF como requerimientos básicos del sistema de salud para alcanzar las metas de control en hipertensión arterial destaca la necesidad de contar con personal entrenado en la toma de la presión arterial como parte de una estrategia de tamizaje adecuada y robusta, un inicio de estrategias de tratamiento tempranas y adecuadas, y un mantenimiento de la adherencia. Además, que se disponga de equipos adecuados para este fin, como esfigmomanómetros avalados y calibrados, y con fácil acceso tanto para el personal de salud como para el paciente, estandarización del recurso intelectual con el desarrollo de guías claras y actualizadas de manejo adecuadas al contexto regional en donde se aplicarán, un sistema de salud organizado de manera tal que permita una fácil interacción de los equipos de salud y la comunidad, que la comunidad conozca lo que significa ser hipertenso y sus implicaciones, y que se tenga en cuenta, por supuesto, el contexto sociocultural del grupo índice para que las estrategias no vayan en contraposición con sus costumbres, financiación del Estado para que el acceso al tamizaje sea gratuito, con intervenciones sostenibles y sistemas de información robustos y confiables, que permitan conocer las cifras de diagnóstico, control y seguimiento para que, de esta manera, se pueda adecuar y optimizar la asignación de recursos y estrategias ${ }^{6}$.

Como conclusión del mapa de ruta planteado por la WHF, todas las naciones deberíamos encaminar nuestras estrategias basándonos en tres puntos principales:

1. Población:

a.Pacientes que no tienen conciencia de sus cifras de presión y sobre quienes es necesario intensificar las estrategias de tamizaje.

b.Pacientes que, conociendo que su presión está elevada, no tienen sus cifras en meta, en quienes se debe favorecer su acceso a estrategias adecuadas de tratamiento.

c. Pacientes que son hipertensos y sus metas están en control, a quienes se debe acompañar con políticas de seguimiento adecuadas para mantener el control.

d. Pacientes que saben que no son hipertensos, a quienes se debe educar en el mantenimiento de estilos de vida saludable y tamizaje frecuente.

2. Tratamiento:

a. Tamizaje oportuno.

b. Tratamiento adecuado.

c. Educación al paciente sobre la importancia del mantenimiento de las estrategias no farmacológicas y farmacológicas (adherencia).

3. Sistema de salud:

a. Tamizaje oportuno.

b. Acceso a tecnologías de salud (medicamentos, tamizaje, procedimientos, etc.).

c. Educación a la comunidad.

d. Telesalud, modelos innovadores de cuidado.

e. Sistemas de información.

En el contexto anterior, en enero de 2020 se desarrolló en Bogotá, Colombia, la mesa redonda (Round Table) de hipertensión arterial, en donde se contó con la presencia de representantes de la WHF en cabeza del Dr. Daniel Piñeiro y un grupo de representantes de cada uno de los grupos que intervienen en el manejo y control de la hipertensión arterial, incluyendo de manera relevante a asociaciones de pacientes para que fueran ellos partícipes de la toma de decisiones, que serán el camino para lograr entre todos un control de esta situación y una mitigación de su impacto nacional. Se contó, pues, con la presencia del ministerio de salud nacional, secretarías de salud, asociaciones académicas, agrupaciones del sector del aseguramiento en salud, facultades de 
medicina, la industria farmacéutica y un grupo de expertos clínicos en el manejo de la hipertensión arterial. Con este valioso capital humano se logró llegar a seis puntos fundamentales de acuerdo, que hoy por hoy trabajamos de manera conjunta para continuar el camino trazado y llegar felizmente a la meta propuesta.

Los puntos de trabajo planteados son los siguientes:

1. Implementación del Registro Nacional de Hipertensión Arterial

Desarrollar un método que permita identificar la incidencia y la prevalencia de la hipertensión arterial en Colombia. Este trabajo permitirá conocer las cifras locales reales y algunas características epidemiológicas de los pacientes afectados por la hipertensión arterial, los cuales serán incluidos en esquemas de prevención dirigida.

2. Estandarización en el método de toma manual de la presión arterial

Dotar a los diferentes centros de atención de la herramienta adecuada para la medición de la presión arterial, con el fin de evitar sesgos en la lectura de la presión y a la vez optimizar los recursos y disminuir su utilización inadecuada. La toma digital deberá estandarizarse en toda la nación, lo cual ayudará a conocer sus números y avanzar en el diagnóstico y el acceso de los pacientes.

3. Estrategia global de educación a la comunidad Creación de estrategias que involucren a la comunidad afectada en el conocimiento de la enfermedad y su implicaciones inmediatas y futuras. Es fundamental educar a pacientes y cuidadores sobre las consecuencias y el impacto metabólico, psicológico y patológico de la hipertensión arterial. De esta manera se generará una mayor conciencia de la enfermedad, el autocuidado y la autogestión del riesgo cardiovascular, y un incremento del compromiso con el manejo no farmacológico.

4. Campaña nacional para disminuir el consumo de alimentos con alto contenido de sodio

Se debe enfocar en un modelo de salud poblacional, orientado a disminuir el consumo de sodio y crear estrategias de comunicación para que las personas conozcan los riesgos de su consumo excesivo.

5. Estrategia global para la detección temprana de la hipertensión arterial

Establecer técnicas de búsqueda e identificación de los pacientes hipertensos para que el curso clínico de cada individuo sea más favorable, y en términos del Estado, de menor costo final. En tal sentido, se disminuye la progresión de la enfermedad a estados terminales.
6. Estrategia global para el acceso a tecnologías de salud en hipertensión arterial

Fomentar políticas públicas que permitan a la comunidad tener acceso a las tecnologías en salud, desde el punto de vista farmacológico, y de estrategias médicas de intervención. Dotar a toda entidad de atención de la tecnología necesaria y adecuada para la medida correcta de la presión arterial.

A la fecha hemos construido puentes adecuados de comunicación entre todos los actores y el ministerio, y se han logrado avances importantes en algunos de estos puntos, permitiéndonos ver y transmitir como mensaje a nuestra comunidad médica que la unidad y el trabajo en grupo son los que marcarán la fortaleza del desarrollo de cada una de las estrategias que las naciones, el continente y el planeta debemos generar en pro del control de esta importante condición clínica.

Hemos iniciado también un proyecto, sin registro previo en Colombia, en la misma línea, generando lazos fuertes entre los actores de los servicios de salud que nos ocupamos en primera mano del manejo de la hipertensión arterial y la prevención cardiovascular. El proyecto PRESIONco consiste en la elaboración de una guía de manejo intersocietaria con un soporte robusto académico, científico y técnico, que incluye a la Sociedad Colombiana de Cardiología y Cirugía Cardiovascular y su capítulo de hipertensión arterial como gestor, con la participación de la Asociación Colombiana de Medicina Interna, la Asociación Colombiana de Endocrinología, la Sociedad Colombiana de Medicina Familiar, la Asociación Colombiana de Nefrología, la Asociación Colombiana de Gerontología y Geriatría, la Universidad Nacional de Colombia y un número importante de especialistas vinculados y colaboradores que seguro tendrá como resultado una unificación de criterios, prácticas y protocolos que se trasladará en beneficio de la población en general.

En conclusión, ante una situación clínica con esta carga tan importante en términos de morbimortalidad y desenlaces cardiovasculares, que se constituye además en una situación relevante de salud pública, se requiere un trabajo conjunto de diferentes instancias, políticas, económicas, académicas, técnicas y sociales, entre otras, para mitigarla e impactarla de manera positiva y lograr, por fin, entre todos, disminuir las cifras relacionadas con la hipertensión arterial.

\section{Financiamiento}

El autor declara que no hay ningún tipo de financiamiento para este artículo editorial. 


\section{Conflicto de intereses}

El autor declara no tener conflicto de intereses.

\section{Responsabilidades éticas}

Protección de personas y animales. El autor declara que para esta investigación no se han realizado experimentos en seres humanos ni en animales.

Confidencialidad de los datos. El autor declara que en este artículo no aparecen datos de pacientes.

Derecho a la privacidad y consentimiento informado. El autor declara que en este artículo no aparecen datos de pacientes.

\section{Bibliografía}

1. Ezzati M, Riboli E. Behavioral and dietary risk factors for noncommunicable diseases. N Engl J Med. 2013;369:954-64.

2. NCD Risk Factor Collaboration (NCD-RisC). Worldwide trends in blood pressure from 1975 to 2015: a pooled analysis of 1479 population-based measurement studies with 19.1 million participants. Lancet. 2017;389:37-55.

3. Moya L, Moreno J, Lombo M, Guerrero C, Aristizábal D, Vera A, et al. Consenso de expertos sobre el manejo clínico de la hipertensión arterial en Colombia. Sociedad Colombiana de Cardiología y Cirugía. Rev Colomb Cardiol. 2018;25:4-26.

4. Chow CK, Teo KK, Rangarajan S, Islam S, Gupta R, Avezum A, et al. Prevalence, awareness, treatment, and control of hypertension in rura and urban communities in high-, middle-, and low-income countries. JAMA. 2013;310:959-68.

5. Olsen MH, Angell SY, Asma S, Boutouyrie P, Burger D, Chirinos JA, et al. $A$ call to action and a lifecourse strategy to address the global burden of raised blood pressure on current and future generations: the Lancet Commission on Hypertension. Lancet. 2016;388:2665-712.

6. Adler AJ, Prabhakaran D, Bovet P, Kazi DS, Mancia G, Mungal-Singh V, et al. Reducing cardiovascular mortality through prevention and management of raised blood pressure: a World Heart Federation Roadmap. Glob Heart. 2015;10:111-22. 